\title{
Morphological Symmetry of Maxillary Anterior Teeth before and after Prosthodontic Planning: Comparison between Conventional and Digital Diagnostic Wax-Ups
}

\author{
Jaafar Abduo \\ Restorative Section, Melbourne Dental School, Melbourne University, Melbourne, Vic., and School of \\ Computer Science and Software Engineering, University of Western Australia, Perth, W.A., Australia
}

\author{
Key Words \\ Dental aesthetics - Digital dentistry $\cdot$ Image registration . \\ Wax-up
}

\begin{abstract}
Objective: The aim of this study was to evaluate the impact of different prosthodontic planning methods on the morphological symmetry of maxillary anterior teeth. Materials and Methods: In 13 patients receiving fixed prosthodontic treatment, dental models were obtained from the Oral Health Centre of Western Australia. Two forms of diagnostic prosthodontic planning were executed: conventional waxup and digital wax-up. The asymmetry was evaluated at two levels: tooth morphological asymmetry (TMA) and anterior segment morphological asymmetry (ASMA). For the TMA, the labial surface of each anterior tooth was superimposed on a mirror image of the contralateral tooth. The ASMA involved superimposition of the combined labial surface of all the anterior teeth on one side on the mirror image of the combined labial surface of the contralateral side. For each asymmetry, the discrepancies in the form of 3D Euclidean distances were calculated and statistically evaluated by the Kruskal-Wallis test $(p=0.05)$. Results: At the TMA level, the digital wax-up models had significantly less asymmetry $(p<$ 0.00 ) than the pre-treatment and conventional wax-up models. The pre-treatment and conventional wax-up models had
\end{abstract}

similar asymmetry $(p=0.35)$. However, at the ASMA level, the difference between the models was insignificant $(p>0.05)$. Conclusion: In this study, the single tooth symmetry had improved after the digital wax-up. However, the conventional wax-up had a minimal impact on single tooth symmetry. ASMA was minimally affected by the two wax-ups.

(c) 2016 S. Karger AG, Basel

\section{Introduction}

Today's population is aesthetically driven and frequently seeks prosthodontic treatment to improve dental appearance [1]. Through individual's life, the teeth may be affected by dental caries, tooth wear and fracture, which will negatively influence their appearance [2]. Prosthodontic treatment, such as crowns, bridges and veneers, has the ability to significantly alter the tooth shape, display and colour [1]. It is acknowledged that the maxillary anterior teeth are the most influential teeth for dental aesthetics, and their presence in harmony is associated with greater aesthetic perception [1]. Despite the debate about which constitutes the ideal dental aesthetics, several variables had been reported $[1,3]$. One of the influential aesthetic variables that should be considered by the treating clinician is dental symmetry. There is agreement that the symmetrical smile is associated with greater aes-

\begin{tabular}{ll}
\hline KARGER & $\begin{array}{l}\text { ( ) 2016 S. Karger AG, Basel } \\
1011-7571 / 16 / 0253-0276 \$ 39.50 / 0 \quad \text { Karger }\end{array}$ \\
$\begin{array}{l}\text { E-Mail karger@karger.com } \\
\text { www.karger.com/mpp }\end{array}$ & $\begin{array}{l}\text { Thisis an Open Access article licensed under the terms of the } \\
\text { Creative Commons Attribution-NonCommercial 3.0 Un- } \\
\text { ported license (CC BY-NC) (www.karger.com/OA-license), } \\
\text { applicable to the online version of the article only. Distribu- } \\
\text { tion permitted for non-commercial purposes only. }\end{array}$
\end{tabular}

Dr. Jaafar Abduo, BDS, DClinDent, MRACDS (Pros), PhD

Melbourne Dental School, Melbourne University

720 Swanston Street

Melbourne, VIC 3010 (Australia)

E-Mail jaafar.abduo@unimelb.edu.au 
thetic perception [4-6]. Therefore, whenever the anterior teeth are restored, dental symmetry should be improved.

Dental symmetry can be evaluated at two levels, symmetry at tooth level and symmetry at arch level. At tooth level, the tooth is symmetrical if it has similar morphology to the contralateral tooth [7]. The arch is deemed symmetrical if the anterior tooth segment on one side is similar to the opposite side. This variable reflects on the symmetry of tooth arrangement and position within the arch, which may influence the perception of dental aesthetics $[6,8]$. From the experimental perspective, the level of asymmetry can be used to measure the deviation from symmetry. Therefore, variables such as tooth morphological asymmetry (TMA) and anterior segment morphological asymmetry (ASMA) can be utilized to measure the deviation from tooth symmetry and arch symmetry, respectively.

To ensure the prosthodontic treatment meets the patient's aesthetic demand, diagnostic wax-up is strongly recommended prior to the definitive treatment [9]. The conventional wax-up involves altering the tooth morphology of the dental cast by addition of wax. Therefore, the diagnostic wax-up simulates the possible dental treatment and improves communication between the patient and the dental technician [9]. Further, the wax-up can be utilized to control tooth preparation and fabrication of provisional restorations $[9,10]$.

With the advancement of digital dentistry, digital waxup has been proposed as a tool for prosthodontic planning [11]. This form of wax-up is based on virtual alterations in the dental morphology. Several methods had been proposed to modify the tooth digitally. This involves the fitting of an average tooth, using a biogeneric library, and mirror imaging of an intact adjacent tooth [7, 12]. It is assumed that the digital alteration of tooth morphology has the advantages of reducing the wax-up time, material manipulation and the number of error-introducing steps. Thus, the digital wax-up is assumed to be more efficient and accurate than the conventional wax-up $[11,13]$. In addition, many software programs allow quantification of the effect of the proposed treatment prior to the active treatment phase. This feature is used to accurately critique the impact of the dental treatment, such as the aesthetic outcome, material thickness and the potential preparation invasiveness $[11,14,15]$. However, a true aesthetic comparison between conventional and digital wax-ups is very rarely reported in the literature $[7,12]$. The purpose of this study was to evaluate the impact of conventional and digital diagnostic wax-ups on the morphological symmetries of the maxillary anterior teeth and arch. The null hypothesis is that the conventional and digital wax-ups could have similar effects on morphological tooth and arch symmetries.

\section{Materials and Methods}

Diagnostic Wax-Ups

Dental models of 13 patients, who required fixed prosthodontic treatment of all the maxillary anterior teeth, were collected. A written informed consent was obtained from each patient. The treatment was indicated to manage failed restorations, unaesthetic teeth or tooth wear (fig. 1a). For all of them, diagnostic wax-up was indicated. The treatments were provided at the Oral Health Centre of Western Australia. Human research ethics approval was obtained from the Human Research Ethics Committee of the University of Western Australia (RA/44/1/5079).

For each patient, an impression of each arch was taken by irreversible hydrocolloid impression material (Alginate; GC America, Alsip, Ill., USA). Type III dental stone (Buff Stone; Adelaide Moulding and Casting Supplies, Somerton Park, S.A., Australia) was used to pour the impressions. Each set of models was duplicated. One set was used for the conventional wax-up and the other for the digital wax-up. The purpose of the wax-up was to restore tooth structures, establish natural tooth morphology, restore symmetry and achieve a physiological occlusion.

For the conventional wax-up, the models were articulated on a semi-adjustable articulator (Whip Mix, Louisville, Ky., USA). The tooth contours were altered by the additive waxing technique, where an inlay wax was added on the external tooth surface by a dental technician with more than 5 years of experience (fig. 1b).

For the digital wax-up, the other set of pre-treatment models was scanned using a microCT scanner (SkyScan; Bruker micro$\mathrm{CT}$, Kontich, Belgium) to obtain digital imaging and communication medicine (DICOM) images. The obtained DICOM images were converted to a virtual 3D stereolithographic model using a DICOM viewing program (CTvox; Bruker microCT; fig. 1c). The digital wax-up was completed by a 3D rendering software package (Geomagic Studio; Raindrop Geomagic Inc., Research Triangle Park, N.C., USA). The opposing models were virtually articulated through the alignment feature of the software. Digital physiological tooth moulds (Phonares Teeth; Ivoclar Vivadent AG, Schaan, Liechtenstein) were used to alter the tooth morphology. Each virtual tooth was manually fitted on the model with the purpose of obtaining an ideal tooth arrangement, emergence profile, symmetry and aesthetics. The virtual tooth alignment process included size alteration, rotation and translation. This was followed by ensuring that adequate occlusal contacts existed (fig. 1d). The digital wax-up was completed by a single operator who is familiar with the software. After the completion of the wax-up, the scanned model and the virtual teeth were merged into a single model, which improved computation speed and the subsequent analysis.

\section{Analysis}

Since the analysis was completed digitally, the conventional wax-up models were converted to virtual models after scanning by the microCT scanner (fig. 1e). The asymmetry was then measured to quantify the deviation from symmetry. Two forms of symmetrical evaluation were applied: TMA and ASMA (fig. 2). The first vari- 

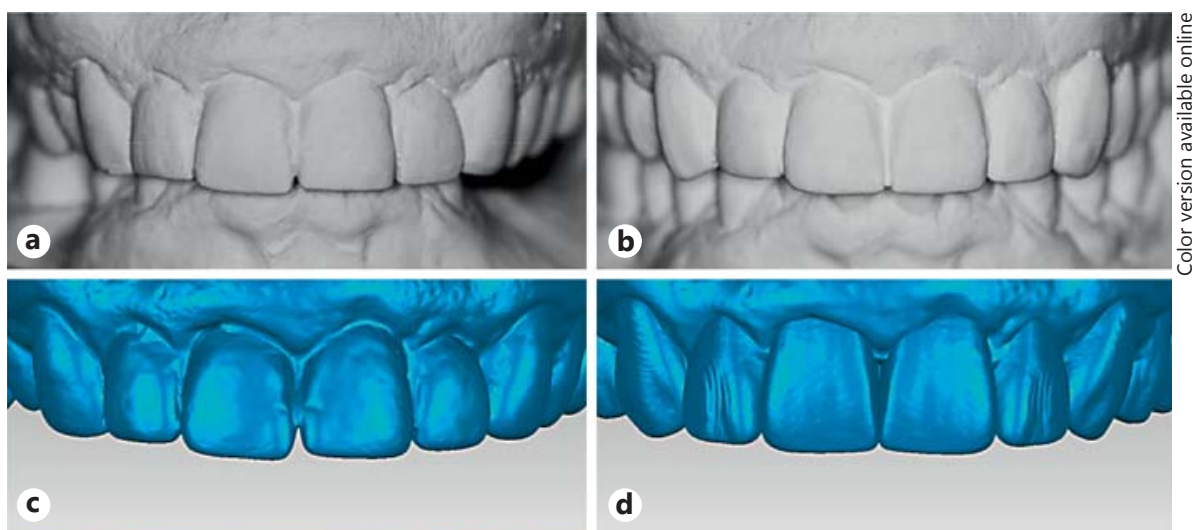

Fig. 1. An example of the diagnostic waxup procedures used to manage anterior dentition discrepancy. a Actual pre-treatment model. b Actual conventional waxup model. c Virtual pre-treatment model. d Digital wax-up model. e Virtual conventional wax-up model.

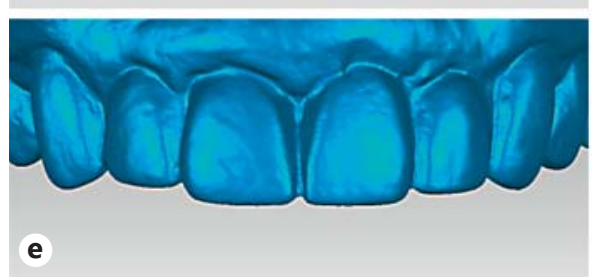

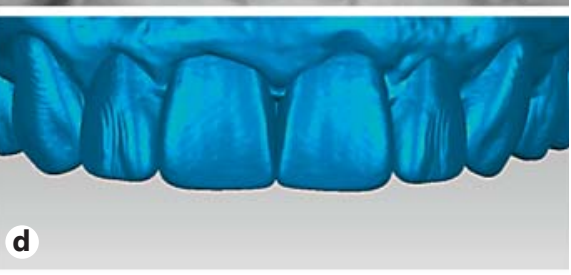

d able provided information about the symmetry improvement of each tooth separately (central incisor, lateral incisor and canine). The ASMA variable was more relevant to the symmetry of the combined tooth shape and position in the arch. For the TMA, the labial surfaces of central incisors, lateral incisors and canines of the pretreatment, conventional wax-up and digital wax-up models were extracted (fig. 2a). The palatal surface was excluded from the experiment since it is largely dependent on the occlusal relationship with the opposing teeth and has minimal impact on symmetry. A mirrored 3D image of the extracted surface of each tooth on the left side was generated and superimposed on the corresponding tooth surface on the right side (fig. $2 \mathrm{~b}, \mathrm{c}$ ). The superimposition process was composed of three sequential steps: point-to-point registration, global registration and the calculation of the 3D Euclidean distances (EDs) [16]. The point-to-point registration determined the initial approximate orientation of the two images by manually locating common anatomical landmarks. The two incisal corners and the most apical cervical region were selected. The global registration was based on the Iterative Closest Point Algorithm, and it aligned the virtual images according to the best-fit principles. The adequacy of the registration was validated by a colour-coded map (fig. $2 \mathrm{~d}$ ). The deviation between two aligned coordinates was determined by the $3 \mathrm{D}$ ED value. The following equation was utilized to calculate the ED between a pre-treatment image point $(\mathrm{P})$ and a corresponding wax-up image point $(\mathrm{W})$ in $3 \mathrm{D}$ coordinates ( $\mathrm{x}, \mathrm{y}$ and $\mathrm{z}$ ).

$$
E D(P, W)=\sqrt{\left(P_{x}-W_{x}\right)^{2}+\left(P_{y}-W_{y}\right)^{2}+\left(P_{z}-W_{z}\right)^{2}}
$$

Eventually, as a function of the software, the deviation between the two images was represented as average 3D EDs of 2,000 random corresponding points on the common surfaces of the superimposed images. Therefore, the lower the mean distance between two images, the better the morphological symmetry.

The ASMA was based on the unilateral extraction of the combined labial surface of central incisors, lateral incisors and canines (fig. 2e). A mirrored image of the left side was generated and superimposed on the right side image. Similar to TMA, the 3D EDs were calculated.

\section{Statistics}

The means and standard deviations for the TMA and ASMA were calculated. To evaluate the presence of a significant difference in the tooth categories and the different models, a series of Kruskal-Wallis tests was implemented $(\mathrm{p}=0.05)$. The Mann-Whitney test was used as a post hoc analysis when a significant difference was identified.

\section{Results}

\section{Tooth Morphological Asymmetry}

The pre-treatment, conventional and digital wax-up models exhibited different TMA. The digital wax-up model had significantly superior symmetry (mean: 31.30 $\pm 15.63 \mu \mathrm{m})$ than pre-treatment (mean: $68.16 \pm 23.33 \mu \mathrm{m})$ and conventional wax-up (mean: $65.77 \pm 27.57 \mu \mathrm{m}$ ) models $(\mathrm{p}<0.00)$. On the other hand, the conventional waxup models had similar symmetry to the pre-treatment models $(\mathrm{p}=0.35)$.

The TMA values for the different tooth categories in all the models are summarised in table 1. Overall, there was similarity in the TMA pattern between the different tooth categories among all the models. The least TMA values were related to the digital wax-up, while the conventional and the pre-treatment models were relatively similar. For each tooth category, the difference between 

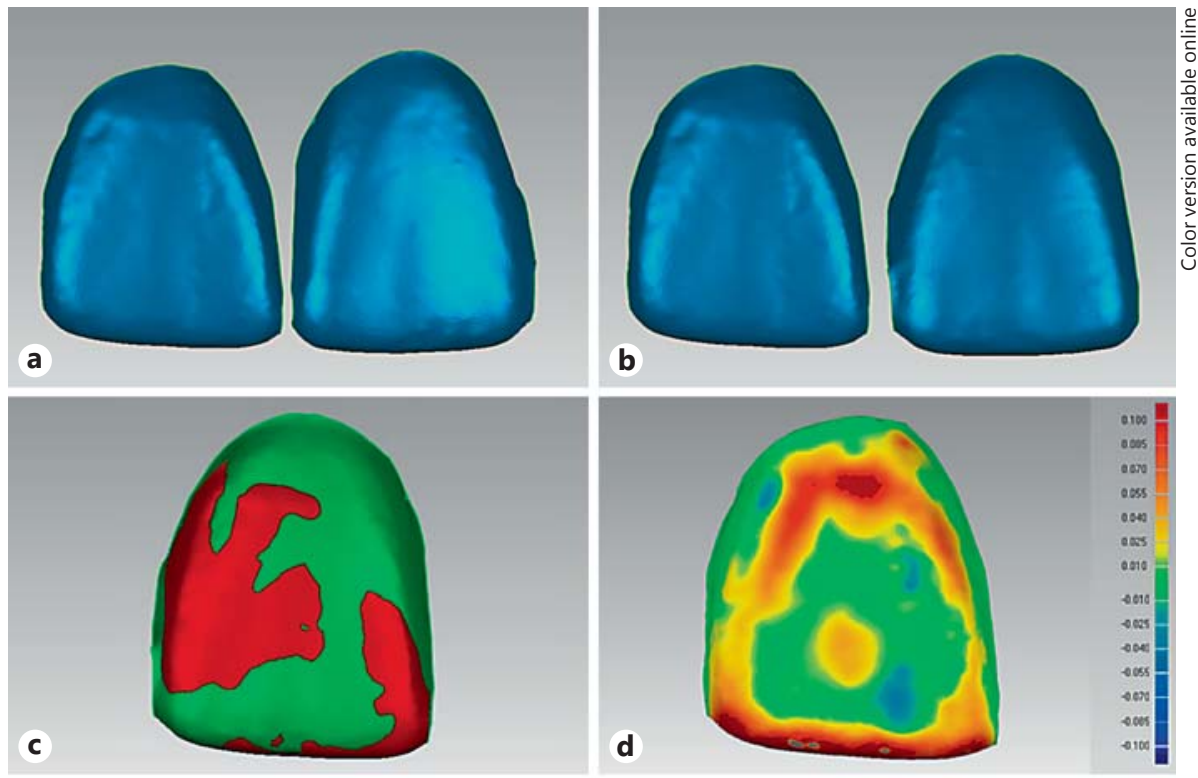

Fig. 2. Examples of the TMA and ASMA evaluation. a Extraction of the labial surfaces of the two central incisors. b The left incisor was converted to a mirror image. c Superimposition process of the two central incisors. d Validation of the superimposition by the colour-coded map. e For the ASMA procedure, the combined labial surface of the central incisor, lateral incisor and canine of one side was extracted.

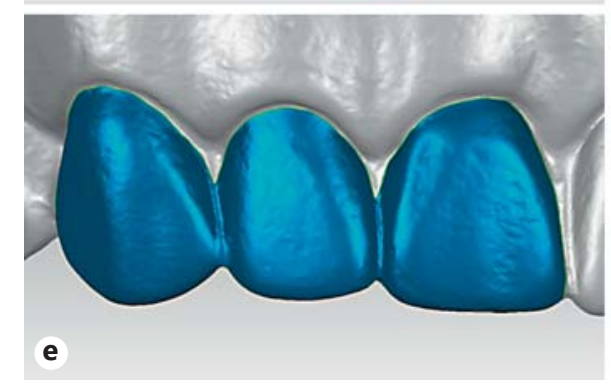

Table 1. TMA for the pre-treatment, conventional wax-up and digital wax-up models (means $\pm \mathrm{SD}$ )

\begin{tabular}{ccll}
\hline & $\begin{array}{l}\text { Pre-treatment } \\
\text { models, } \mu \mathrm{m}\end{array}$ & \multicolumn{2}{l}{ Wax-up models, $\mu \mathrm{m}$} \\
\cline { 3 - 4 } & & & \\
\hline conventional & digital \\
\hline Incisors & & $59.89 \pm 17.57$ & $25.48 \pm 11.20$ \\
Central & $67.12 \pm 21.43$ & $60.23 \pm 25.14$ & $31.33 \pm 16.78$ \\
Lateral & $57.65 \pm 18.48$ & $77.20 \pm 35.42$ & $37.08 \pm 17.13$ \\
Canines & $79.72 \pm 25.63$ & & \\
\hline
\end{tabular}

the digital wax-up models and the pre-treatment and conventional wax-up models was significant $(\mathrm{p}=0.00)$. However, the conventional wax-up and pre-treatment models had statistically similar TMA for the central incisors $(\mathrm{p}=0.43)$, lateral incisors $(\mathrm{p}=0.90)$ and canines $(\mathrm{p}=0.54)$.

After comparing the different teeth, there was a tendency for the canines to exhibit the greatest TMA. This pattern was observed in the pre-treatment, conventional wax-up and digital wax-up models. However, the statistically significant difference existed only between the canines and the lateral incisors of the pre-treatment models $(p=0.02)$. The difference was insignificant between the canines and the central incisors $(\mathrm{p}=0.63)$ or the lateral incisors $(p=0.13)$ for the conventional wax-up. Similarly, for the digital wax-up, there was an insignificant difference between the canines and the central incisors ( $\mathrm{p}=$ $0.09)$ or lateral incisors $(\mathrm{p}=0.32)$.

\section{Anterior Segment Morphological Asymmetry}

The mean ASMA values for the pre-treatment, conventional wax-up and digital wax-up models were $203.44 \pm 78.04,148.21 \pm 41.26$ and $162.28 \pm 48.97 \mu \mathrm{m}$, respectively. The pre-treatment models had insignificantly greater ASMA values than the wax-up models. The two wax-up models had relatively similar ASMA $(\mathrm{p}>0.05)$. 


\section{Discussion}

In this study, the tooth morphological symmetry had improved after the wax-ups; however, the improvement was more prominent for the digital wax-up than conventional wax-up. On the other hand, the morphological symmetry of the arch was minimally affected by the waxups. Although diagnostic wax-up improved tooth morphology and appearance, the discrepancy between the tooth level and the arch level morphological symmetries was attributed to the inability of the prosthodontic treatment to alter the position of the tooth within the arch.

According to the TMA values, it appeared that there was a significant advantage of the digital wax-up. Therefore, the hypothesis that the conventional and digital wax-ups will have similar effect on morphological tooth symmetry is rejected. The superiority of digital wax-up is related to the mathematical determination of the tooth morphology. Through the process of digital wax-up, an average tooth shape is selected and fitted on the tooth that will be modified $[7,12]$. Further, the mirrored image feature is implemented [7]. This feature was shown to be a significant benefit for digital modelling of teeth $[7,12]$ and facial tissues [17] since it accurately establishes the contralateral morphology. On the contrary, the conventional wax-up is vulnerable to more human intervention, which explains the greater tooth morphological asymmetry in comparison to the digital wax-up models. While in many cases the obtained conventional wax-up outcome is deemed aesthetic, to a certain degree, symmetry discrepancy is inevitable due to great reliance on the artistic abilities of the dental technician. In accordance with the outcome of this study, Vasconcelos et al. [18] had evaluated tooth contour alterations with composite veneer fabricated by three dental technicians. The technicians were requested to fabricate the veneers with and without the presence of an anatomical reference tooth. Even with the presence of the anatomical reference tooth contour, an increase in the contour of up to $0.5 \mathrm{~mm}$ was observed. This indicates that even if the desired morphology is available as a guide for the technician, obtaining exactly similar morphology is very difficult by manual contouring. Achieving symmetrical outcome by the conventional method is further complicated by the susceptibility of wax to temperature-related dimensional distortion [14].

Although the digital wax-up had statistically greater morphological tooth symmetry than the conventional wax-up, the actual difference between the two wax-ups was minimal $(<0.10 \mathrm{~mm})$, which will be hardly noticeable clinically as several studies had concluded that lay per- sons cannot detect symmetrical differences in the range of $0.5-2 \mathrm{~mm}[4,5]$. In nature, slight asymmetry is common within natural and non-restored dentition. Mavroskoufis and Ritchie [19] had found that $60 \%$ of young individuals had an accumulated central incisors discrepancy of more than $0.2 \mathrm{~mm}$. Likewise, a minimal magnitude of dimensional difference between the opposing maxillary anterior teeth was observed by Gillen et al. [3].

In relation to the ASMA values, the two wax-ups are similar and they did not significantly improve the ASMA of the pre-treatment models. Thus, the hypothesis that the two wax-ups will have similar effect on arch symmetry is accepted. As this variable reflects the positional symmetry, it can be speculated that the final arch morphological symmetry is largely dependent on the initial tooth position and curvature of the arch $[1,20]$ rather than the impact of the prosthodontic treatment. Therefore, although the fixed prosthodontic treatment can improve the tooth morphology, it will minimally influence the positional symmetry. To improve the positional symmetry, adjunctive treatment might be necessary. Nevertheless, it is important to note that although dental symmetry is one of the important aesthetic variables [6], its impact on the aesthetic perception has not been fully quantified. On the other hand, several studies have reported that minor deviation in the symmetry is not necessarily noticeable by lay persons. In an aesthetic appraisal, it was found that $0.5-1.0 \mathrm{~mm}$ vertical discrepancies of the lateral incisors were hardly detectable by lay persons [5]. Similarly, Pinho et al. [4] had found that even a 2-mm perceived asymmetry of the canine cusp tip was acceptable by lay persons. Thus, reasonable deviation from absolute symmetry is not necessary perceived as unaesthetic.

Among all the models, the TMA pattern indicated that the canines tend to have the greatest asymmetry. This is most likely related to the position of the canines in relation to the midline [1]. Several reports had indicated the importance of symmetry for the teeth closer to the midline. As the distance increases from the midline, the need for optimal symmetry is reduced. For example, Brunzel et al. [8] had shown that the symmetrical position of the central incisors is crucial while a minor discrepancy in the lateral incisors position can be tolerated. Furthermore, discrepancies at the canines were found to be more acceptably perceived than other anterior teeth [4]. Therefore, it is likely that the operator will aim to improve the symmetry of the central incisors followed by the lateral incisors and the canines, respectively. Anatomically, the clinical crown of a canine tooth has two labial surfaces: a mesial surface that is more noticeable from the frontal perspective and a 
distal surface that can be observed by a buccal view. As a result, the total canine morphology can tolerate more alterations, by increasing or decreasing the tooth width, yet without significantly affecting the frontal view.

Although digital wax-up was advantageous in many aspects, it still needs validation. The observation of this study was based on digital image registration which quantifies the similarity between two surfaces [16] and does not determine the true aesthetic value. Future experiments should be aimed at measuring the patient's satisfaction level after each wax-up to confirm the aesthetic benefit of the digital stream. On the other hand, the digital wax-up should be practical, cost effective and time efficient. For example, for the digital wax-up to be applicable, it should be transferrable to the clinic. Physical models of the digital wax-up can only be produced by $3 \mathrm{D}$ printing or milling [21], which will introduce actual discrepancies. For instance, dimensional error of models produced by 3D printing can be greater than $100 \mu \mathrm{m}$ [22]. Similarly, Cho et al. [23] had found that dental casts produced by conventional methods exhibited better overall accuracy than digitally produced casts. The implications of computerized production on the digital wax-up have to be investigated in further research.

\section{Conclusion}

This study illustrates the merit of using digital technologies in obtaining dental morphologies. In comparison with the conventional wax-up, the digital wax-up was associated with greater single tooth symmetry than the conventional wax-up. On the other hand, the anterior segment symmetry was minimally affected by the two wax-ups. Therefore, although the prosthodontic treatment is known to improve aesthetics by changing the tooth anatomy and shade, the improvement in morphological symmetry was limited.

\section{Acknowledgements}

This experiment was supported by a Research Development Award from the University of Western Australia. The author acknowledges the National Imaging Facility at the Centre for Microscopy, Characterization and Analysis, University of Western Australia. The author would like to thank the staff at the technical facility of the Oral Health Centre of Western Australia.

\section{References}

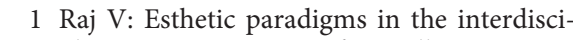
plinary management of maxillary anterior dentition - a review. J Esthet Restor Dent 2013;25:295-304.

2 Lucas PW, van Casteren A: The wear and tear of teeth. Med Princ Pract 2015;24(suppl 1): 3-13.

- 3 Gillen RJ, Schwartz RS, Hilton TJ, et al: An analysis of selected normative tooth proportions. Int J Prosthodont 1994;7:410-417.

4 Pinho S, Ciriaco C, Faber J, et al: Impact of dental asymmetries on the perception of smile esthetics. Am J Orthod Dentofacial Orthop 2007;132:748-753.

5 Machado AW, Moon W, Gandini LG Jr: Influence of maxillary incisor edge asymmetries on the perception of smile esthetics among orthodontists and laypersons. Am J Orthod Dentofacial Orthop 2013;143:658-664.

6 Durgekar SG, K N, Naik V: The ideal smile and its orthodontic implications. World J Orthod 2010;11:211-220.

7 Probst FA, Mehl A: CAD reconstruction using contralateral mirrored anterior teeth: a 3 -dimensional metric and visual evaluation. Int J Prosthodont 2008;21:521-523.

8 Brunzel S, Kern M, Freitag S, et al: Aesthetic effect of minor changes in incisor angulation: an internet evaluation. J Oral Rehabil 2006;33: 430-435.
-9 Magne P, Belser UC: Novel porcelain laminate preparation approach driven by a diagnostic mock-up. J Esthet Restor Dent 2004;16:7-16.

10 Gurel G: Porcelain laminate veneers: minimal tooth preparation by design. Dent Clin North Am 2007;51:419-431.

-11 Abduo J, Bennamoun M, Tennant M, et al: Precision of digital prosthodontic planning for oral rehabilitation. Br J Appl Sci Technol 2014;4:3915-3929.

12 Mehl A, Blanz V, Hickel R: Biogeneric tooth: a new mathematical representation for tooth morphology in lower first molars. Eur J Oral Sci 2005;113:333-340.

13 Beuer F, Schweiger J, Edelhoff D: Digital dentistry: an overview of recent developments for $\mathrm{CAD} / \mathrm{CAM}$ generated restorations. Br Dent J 2008;204:505-511.

14 Abduo J, Lyons K, Bennamoun M: Trends in computer-aided manufacturing in prosthodontics: a review of the available streams. Int J Dent 2014;2014:783948.

15 Davis GR, Tayeb RA, Seymour KG, et al: Quantification of residual dentine thickness following crown preparation. J Dent 2012;40: 571-576.

16 Abduo J, Bennamoun M: Three-dimensional image registration as a tool for forensic odontology: a preliminary investigation. Am J Forensic Med Pathol 2013;34:260-266.
17 Feng ZH, Dong Y, Bai SZ, et al: Virtual transplantation in designing a facial prosthesis for extensive maxillofacial defects that cross the facial midline using computer-assisted technology. Int J Prosthodont 2010;23:513-520.

18 Vasconcelos FS, Neves AC, Silva-Concilio LR, et al: Influence of anatomic reference on the buccal contour of prosthetic crowns. Braz Oral Res 2009;23:230-235.

19 Mavroskoufis F, Ritchie GM: Variation in size and form between left and right maxillary central incisor teeth. J Prosthet Dent 1980;43: 254-257.

20 Ward DH: Proportional smile design using the recurring esthetic dental (red) proportion. Dent Clin North Am 2001;45:143-154.

21 Kasparova M, Grafova L, Dvorak P, et al: Possibility of reconstruction of dental plaster cast from 3D digital study models. Biomed Eng Online 2013;12:49.

22 Inokoshi M, Kanazawa M, Minakuchi S: Evaluation of a complete denture trial method applying rapid prototyping. Dent Mater J 2012; 31:40-46.

23 Cho SH, Schaefer O, Thompson GA, et al: Comparison of accuracy and reproducibility of casts made by digital and conventional methods. J Prosthet Dent 2015;113:310-315. 